\title{
QUADRICUSPID AORTIC VALVE
}

\author{
Aurelian Corneliu Moraru ${ }^{1,2}$, Ioana Maria Ceomîrtan-Negrii ${ }^{1,3}$, Mariana Floria ${ }^{1,3}$ \\ "'Dr. Iacob Czihac" Military Emergency Clinical Hospital Iași; \\ ${ }^{2}$ Romanian Academy; \\ ${ }^{3}$ Grigore T. Popa University of Medicine and Pharmacy Iași; \\ Corresponding author: \\ Ioana Maria Ceomîrtan-Negrii, "Grigore T. Popa" University of Medicine and Pharmacy, 16 \\ University Street, 700115, Iași, Romania \\ Email: ioana_ceomirtan@yahoo.com
}

Keywords: quadricuspid aortic valve, transthoracic bidimensional echocardiography.

Quadricuspid aortic valve (QAV) is a rare congenital anomaly consisting in one supernumerary cusp. It is frequently associated with aortic regurgitation, symptomatic high blood pressure and other congenital defects, being usually diagnosed in the fifth or sixth decade of life ${ }^{(1,2)}$.

Case presentation. A 34-year old man was admitted for high normal/borderline blood pressure. Without previous medical history, he complained about progressive shortness of breath. Transthoracic bidimensional echocardiography revealed QAV type I or A (Figure 1); maximum and mean aortic valve gradient was 23 and $15 \mathrm{mmHg}$, respectively; ascending aorta diameter of $42 \mathrm{~mm}$ (20.48 $\left.\mathrm{mm} / \mathrm{m}^{2} ; \mathrm{N} \leq 1.7 \mathrm{~cm} / \mathrm{m}^{2}\right)$; planimetric aortic valve area of $3 \mathrm{~cm}^{2}$; moderate central aortic regurgitation (PHT of $610 \mathrm{~ms}$, vena contracta of $5 \mathrm{~mm}$ ); mild left ventricular hypertrophy without dilatation; normal left ventricular systolic and diastolic function, mild left atrial dilation $\left(36 \mathrm{ml} / \mathrm{m}^{2}\right)$. No other congenital defects or signs of pulmonary hypertension were detected.

Ambulatory blood pressure monitoring revealed mildly blood pressure elevated values. Therefore, cardiac risk factors correction and an angiotensin converting enzyme drug were recommended.

Discussion. QAV development might be due to the abnormal septation of embryological arterial

trunk, to the aberrant fusion of the aorticopulmonary septum or to the abnormal proliferations in the common trunk ${ }^{1-3}$. The incidence of QAV, which is slight male predominant, is about $0.7 \%^{(1)}$; other congenital heart defects can be present in 18$32 \%$ of these patients (coronary artery and coronary ostium anomalies, atrial or ventricular septal defect, patent ductus arteriosus, etc $)^{(1)}$. There are two classification for QAV based on the relative size (from $\mathrm{A}$ to $\mathrm{H}$ type; Figure $2 \mathrm{~A}$ ) and on the position (from I to IV type; Figure 2B) of the supernumerary cusp $^{(1)}$. Progressive cusp fibrosis with subsequent failure of cusp coaptation, 


\section{INTERA}

unequal distribution of stress and incomplete coaptation of the cusps are the main mechanisms of aortic regurgitation appearance and progression associated with $\mathrm{QAV}^{(1-3)}$.

Echocardiography examination reveals a crosslike appearance in diastole and a squarelike appearance in systole. Transesophageal echocardiography, cardiac computer tomography and cardiac magnetic resonance imaging represent more accurate imaging for diagnosis of QAV in asymptomatic patients. Appropriate followup with antibiotic prophylaxis against infective endocarditis is mandatory in the case of QAV with unequal-sized cusps. More than $50 \%$ of patients with QAV will undergo surgical intervention aiming to correct the aortic regurgitation in symptomatic patients with left ventricular dysfunction and remodeling $^{(1-3)}$. The optimal technique for QAV repair is considered to be tricuspidalization, although aortic valve replacement can be used $^{(1)}$. Patient selection and surgical procedure type are very important, as not all patients with QAV require surgical correction $^{(1,2)}$. Patient prognosis depends on, cardiac risk factors, associated comorbidities and surgical treatment type.

\section{References}

1. Veronese $E T$, Brandão CMA, Steffen SP, Pomerantzeff $P$, Jatene FB. Quadricuspid Aortic Valve: Three Cases Report and Literature Review. Braz J Cardiovasc Surg 2019;34(5):637-639.

2. Yuan SM. Quadricuspid Aortic Valve: A Comprehensive Review. Braz J Cardiovasc Surg 2016;31(6):454-460.

3. Vasudev $R$, Shah $P$, Bikkina $M$, Shamoon $F$. Quadricuspid Aortic Valve: A Rare Congenital Cause of Aortic Insufficiency. J Clin Imaging Sci 2016;6:10. 


\section{in Medicine}

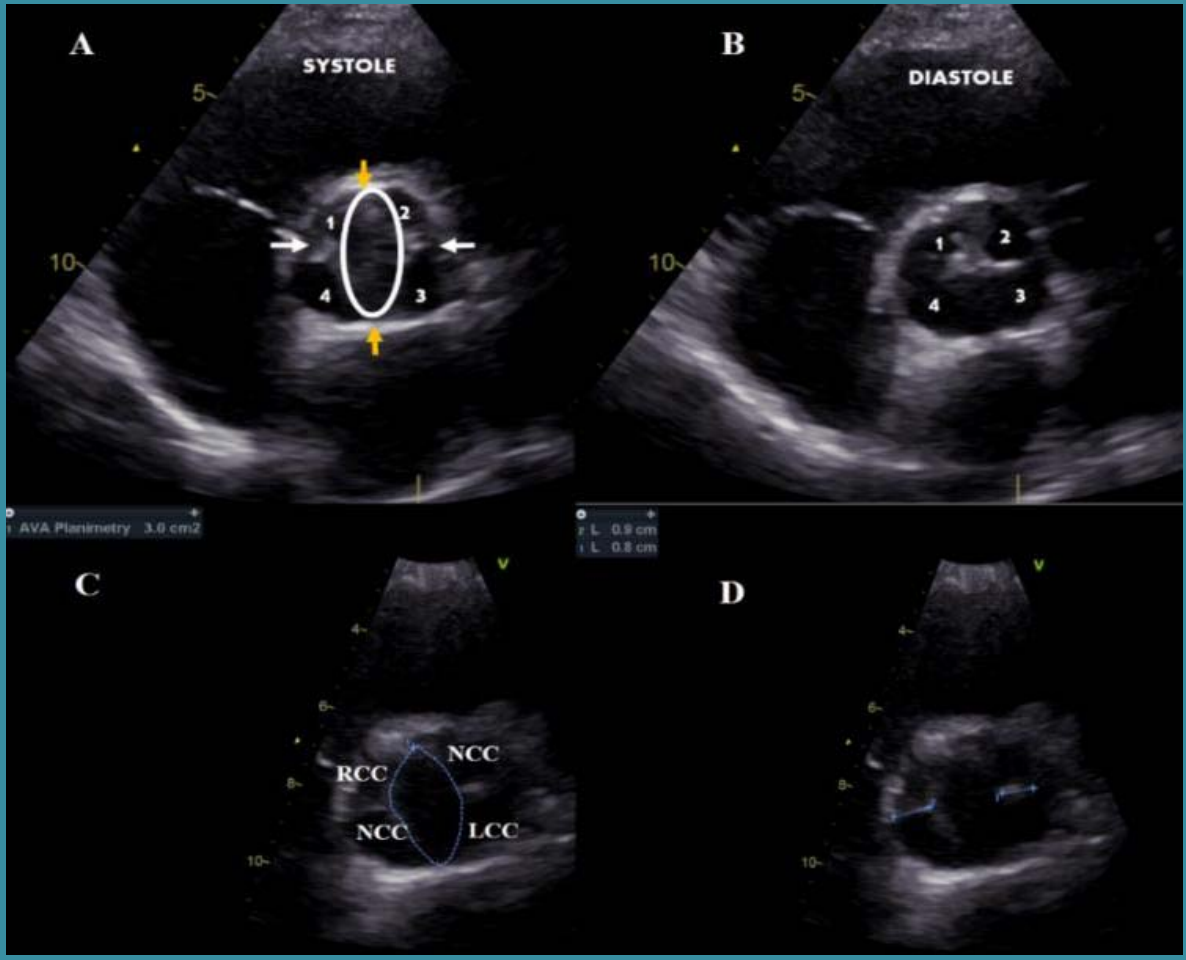

Figure 1. Transthoracic echocardiography parasternal short axis view in systole and diastole with the four cusps: right coronary cusp (RCC), left coronary cusp (LCC) and two non-coronary cusp (NCC).

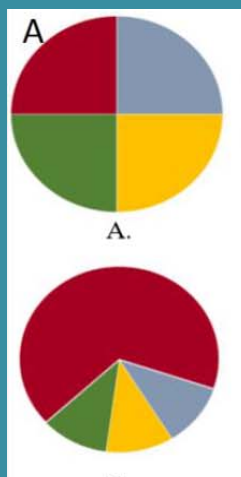

E.

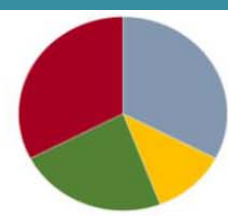

B.

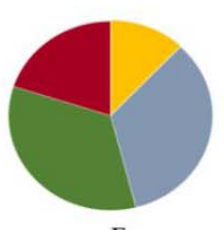

F.

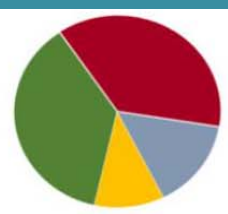

C.

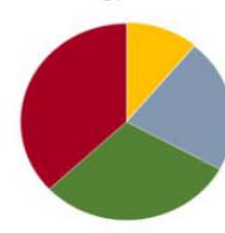

G.

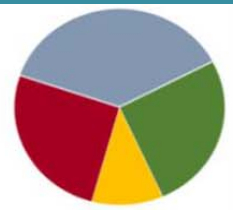

D.

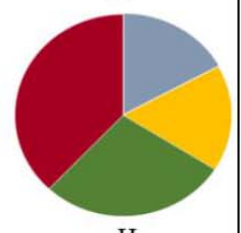

H.

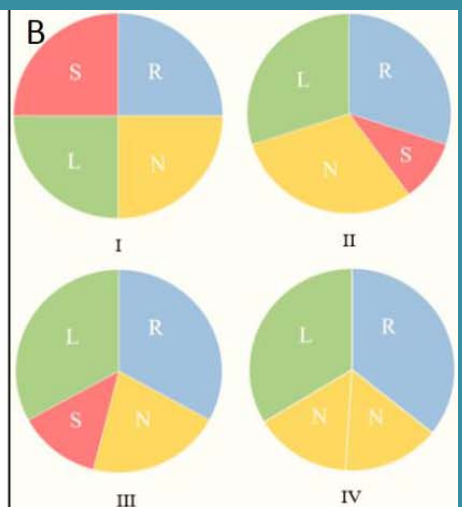

IV

Figure 2. The classification of the quadricuspid aortic valve. $2 A$ : based on the relative size of supernumerary cusp (from A to $H$ type); $2 B$ : based on the position of the supernumerary cusp (from I to IV type). L: left cusp; N: non-coronary cusp; R: right cusp; S: supernumerary cusp. 